\title{
Parámetros poblacionales de Tuta absoluta (Lepidoptera: Gelechiidae) $y$ pérdidas asociadas en tomate de invernadero
}

José Alexander Pérez Marulanda ${ }^{1}$ \& Carlos Eduardo Giraldo Sánchez ${ }^{2 *}$

1. Semillero BIOECO, Universidad Católica de Oriente, Sector 3 Cr 46 \# 40B-50, Rionegro, Antioquia, Colombia; jolerez.92@gmail.com

2. Grupo de Investigación de Sanidad Vegetal, Universidad Católica de Oriente, Sector 3 Cr 46 \# 40B-50, Rionegro, Antioquia, Colombia; cegiral0@gmail.com

* Correspondencia

Recibido 28-II-2020. Corregido 08-VII-2020. Aceptado 14-VII-2020.

\begin{abstract}
Population parameters of Tuta absoluta (Lepidoptera: Gelechiidae) and associated losses in greenhouse tomato. Introduction: The tomato leafminer, Tuta absoluta (Meyrick), the most threatening pest for tomato, Solanum lycopersicum L., production worldwide, may cause total losses, and is difficult to control. Thus, understanding its populations dynamic in the crop is a priority to mitigate damage. Objective: Herein, population parameters of T. absoluta were evaluated in tomato var. Sonero in the greenhouse, in three management systems (commercial, maximum population and total control). Methods: during 25 weeks in La Union, Antioquia, Colombia, mathematical models were applied to describe the populations of larvae and adults (males) in time, as related to production parameters and associated losses. Results: The intrinsic growth rate was 0.447 larvae per week. The Gaussian and exponential models described best the population over time. The losses were associated with weight and number of marketable fruits, and with total production weight, and number of clusters and total fruits. Conclusion: T. absoluta larvae have growth exponentially in a ventilated greenhouse, with a smaller intrinsic growth rate than that calculated by life tables in the laboratory.
\end{abstract}

Key words: damage index; tomato leafminer; tomato moth; Integrated Pest Management; exponential growth.

Pérez Marulanda, J.A., \& Giraldo Sánchez, C.E. (2020). Parámetros poblacionales de Tuta absoluta (Lepidoptera: Gelechiidae) y pérdidas asociadas en tomate de invernadero. Revista de Biología Tropical, 68(4), 1025-1038.

La polilla del tomate, Tuta absoluta (Meyrick) (Lepidoptera: Gelechiidae) ha expandido su distribución original en Suramérica a Europa (Desneux et al., 2010; Van der Straten, Potting, \& van der Lindena, 2011; Araya, Hernando, \& Fereres, 2017) y África (Guimapi et al., 2016; Zekeya et al., 2017a; Zekeya, Ndakidemi, Chacha, \& Mbega, 2017b), donde causa pérdidas de hasta 80-100 \% (Cocco, Serra, Lentini, Deliperi, \& Delrio, 2015). Esta plaga amenaza a la producción mundial (Desneux et al., 2011), y se requieren estrategias que ayuden a mitigar sus daños (FAO, 2016; Marchioro, Krechemer, \& Foerster 2017). Las larvas afectan el follaje, flores y frutos, e incluso causan la muerte de plantas jóvenes, y grandes pérdidas en rendimiento (Mohamed, Mohamed, \& Gamiel, 2012; Goftishu, Seid, \& Dechassa, 2014). Actualmente, el control más empleado se enfoca en pesticidas de síntesis química de amplio espectro, generalmente, sin usar criterios como el Nivel de Daño Económico (NDE) para la plaga (Balzan \& Moonen, 2012). El uso continuo 
e indiscriminado de estas moléculas han generado la aparición de poblaciones resistentes en todo el mundo (Salazar \& Araya, 2001; Silva et al., 2015; Roditakis et al., 2018).

Se han evaluado modelos que relacionan la temperatura y el desarrollo de T. absoluta (Krechemer \& Foerster, 2015; Özgökçe, Bayindir, \& Karaca, 2016; Marchioro et al., 2017). Sin embargo, poco se ha realizado al respecto de relacionar estas modificaciones con las poblaciones en campo en cultivos comerciales. Los modelos matemáticos se usan para identificar procesos determinantes en la dinámica de una población (Sharma Abbott, Dutta, \& Gupta, 2015; Nguyen \& Nansen, 2018). Entre los más usados para estudiar poblaciones están el logístico y el exponencial. El primero evalúa el equilibrio en la población, o capacidad de carga (K) (Sharma et al., 2015). El crecimiento exponencial es apropiado para estudiar poblaciones en periodos cortos de tiempo, y permite la predicción de umbrales sobre insectos plaga (Vandermeer, 2010). Para T. absoluta, se han desarrollado aproximaciones recientes para modelar el incremento de sus poblaciones en laboratorio (Kanle-Satishchandra, Chakravarthy, Özgökçe, \& Atlihan, 2019). Sin embargo, aún no se han relacionado los parámetros obtenidos en laboratorio, con datos obtenidos de poblaciones naturales en cultivos comerciales con y sin control de plagas.

Este estudio estimó la tasa intrínseca de crecimiento (r) y la población máxima (Nf), tanto en larvas como en machos adultos, evaluó los modelos matemáticos que mejor describen el comportamiento de la población en el tiempo, y estimó las pérdidas asociadas a la plaga con parámetros del cultivo bajo tres sistemas de manejo, calculando el índice de daño (ID) para cada uno de los parámetros evaluados.

\section{MATERIALES Y MÉTODOS}

Localización: El trabajo se desarrolló en un área de bosque montano bajo muy húmedo (bmh-MB) (Holdridge, 1967) en el Municipio de La Unión, Antioquia, Colombia (5 ${ }^{\circ} 95^{\prime} 8.87^{\prime \prime}$ N \& 75³7’3.34” E), a 2464 m.s.n.m. brillo solar de $200 \mathrm{~h}$ al año, $2500-3000 \mathrm{~mm}$ precipitación promedio anual y $96 \% \mathrm{HR}$.

Establecimiento del cultivo, labores culturales y unidades experimentales: Se estableció un invernadero de $300 \mathrm{~m}^{2}(10 \times 30 \mathrm{~m})$ con altura máxima de $4 \mathrm{~m}$ y lateral de $2.30 \mathrm{~m}$, con ventilación cenital, frontal y lateral. Se regó por goteo dos veces al día, siguiendo a Flores, Ojeda-Bustamante, López, Rojano, \& Salazar, 2007).

El ensayo se dividió en tres sistemas de manejo de plagas, comercial, máxima población de T. absoluta y control total, los cuales se aislaron entre ellos para evitar el vuelo de polillas entre los mismos. El sistema comercial, de $260 \mathrm{~m}^{2}$ y 590 plantas, estaba abierto a la entrada de la plaga desde el exterior; los sistemas de máxima población y control total tuvieron cada uno $20 \mathrm{~m}^{2}$ y 40 plantas; en ambas áreas cada planta se identificó para los muestreos. Estos sistemas se aislaron con malla antitrips de 20 x 10 hilos $/ \mathrm{cm}^{2}$, de 2.20 x 4 m de largo x $5 \mathrm{~m}$ de ancho, y un techo de 16 x 6 m. Ambas áreas tuvieron puertas de $2 \times 0.8 \mathrm{~m}$, y se aislaron con malla antitrips. Los sistemas se identifican en adelante como "Comercial", "Tuta" y "Cero".

Se plantó tomate var. Sonero, a una densidad de 2.23 plántulas $/ \mathrm{m}^{2}$, que se amarraron a tutores verticales, siguiendo a Pihán y Marín (2000). Se hizo poda de formación 23 días después de la siembra (dds), y de brotes desde los 30 dds, 2 veces por semana. Se hizo podas sanitarias a los 103 y 136 dds. Las plantas se aporcaron a los 15 dds y las malezas se arrancaron cada $15 \mathrm{~d}$. Se abonó N, P, K al suelo, follaje y por fertirriego; $5 \mathrm{~g} /$ planta (18-46-0) en la semana 2 y durante la etapa vegetativa; $20 \mathrm{~g} /$ planta (10-20-20) cada 20 días en floración y finalización. El fertirriego aportó semanalmente 1000 $\mathrm{g}$ de $\mathrm{CaO}+\mathrm{B}$ y $500 \mathrm{~g}$ de $\mathrm{MgO}$, desde la semana 6 después del cuajado de frutos. Las aplicaciones foliares contra patógenos se hicieron según las necesidades del cultivo. Se aplicó en presiembra Trichoderma asperellum, T. atroviride y T. harzianum, y luego aplicaciones foliares de Difenoconazole, Mancozeb, Metalaxyl-M - Mancozeb, Carbamato + Benzamida, Sulfato 
de Gentamicina + Clorhidrato de oxitetraciclina, Azoxystrobin + Difeconazole, Myclobutanil, Kasugamicina, Fluopyram + Pyrimethanil. El manejo de plagas en el sistema comercial se hizo con una rotación por ingrediente activo de Lambda-cihalotrina, Cipermetrina, Sulfoxaflor, Thiamethoxam - Lambda-cihalotrina, Spinetoram y Benzoato de emamectina, en las semanas $10,12,14$ y 23. En cada mezcla se agregó coadyuvante de Polioxyethylene - Polimero estirenado. Los insecticidas contra $T$. absoluta no se hicieron en el sistema Tuta y en el sistema cero se hizo erradicación manual de cualquier insecto plaga.

En los tres sistemas de manejo, se seleccionaron 10 plantas y se registró semanalmente la altura, diámetro del tallo en el cuello, distancia ente racimos, desde el trasplante hasta la semana 25; y en la floración desde la semana 16 hasta la cosecha final, se registraron el número y peso de los frutos, por racimo y por planta.

Se anotó la temperatura máxima y mínima y la humedad con un termohigrómetro digital (Acurite) ref-411108 en el sistema Tuta, y un termómetro digital ref-TFA 30.1039 en el área comercial. La precipitación se registró con un pluviómetro digital (Acurite) ref-00896, con recolector de auto vaciado, a $9.72 \mathrm{~m}$ del invernadero. Los registros se hicieron después del trasplante, semanalmente, durante 30 semanas.

Para el sistema Tuta se capturaron 50 larvas de T. absoluta de último estadio, en cultivos comerciales vecinos al invernadero, que se criaron en cajas de $20 \times 40 \times 15 \mathrm{~cm}$ para obtener adultos, de los que 5 parejas de adultos sexados se liberaron, una semana después del trasplante de las plántulas.

Tuta absoluta: Una semana después del trasplante se comenzó el muestreo en 40 plantas en los tres sistemas y se registró el número de larvas por planta desde la semana uno hasta la 15 dds; después (semanas 16 a 30), el muestreo se hizo en 10 plantas por sistema. El muestreo en el sistema Tuta finalizó en la semana 25 tras la cosecha, dado que las plantas comenzaron a senescer en la semana 21; en los otros dos sistemas, el muestreo se extendió hasta la semana 30. Para evaluar las larvas en las secciones media, superior y apical se seleccionaron 10 plantas que se marcaron; los registros fueron semanales y las secciones se ajustaron según el crecimiento de las plantas.

En el sector Comercial se hizo captura masiva y muestreo de machos con una trampa plástica de $4 \mathrm{~L}$, con ventanas frontales de $12 \mathrm{x}$ $6 \mathrm{~cm}$, feromona (3E,8Z,11Z)-3,8,11-tetradecatrien-1-il acetato y (3E,8Z)-3,8-tetradecadien1-il acetato), a $789.89 \mathrm{mg}$ ia./Kg de producto (Safer, 2017) en un dispersor sujeto con alambre en el extremo superior, y $10 \mathrm{~mL} / \mathrm{L}$ de agua jabonosa en el fondo. Esta trampa se instaló dentro del invernadero, a $1.4 \mathrm{~m}$ de distancia del borde y $10 \mathrm{~cm}$ del suelo. El registro de capturas se inició desde la tercera semana dds, durante 30 semanas, con renovación semanal de la solución jabonosa.

En el sistema Tuta se utilizó una trampa similar en la semana 26 , durante $30 \mathrm{~d}$, con recuentos diarios y remoción de los machos capturados.

Análisis de datos y modelación estadística: Los resultados se evaluaron con modelos lineales (Cook, 1977; Rencher \& Schaalje, 2008) y no lineales (Brown, \& Rothery, 1993; Gaussiano, Brown, \& Rothery, 1993; Franceschetti \& Riccio, 2007). Los modelos se evaluaron bajo el criterio de información Akaike (AIC) (González, Lise, \& Felpeto, 2013). Para los modelos se construyeron intervalos de confianza del $95 \%$, estimados mediante 1999 réplicas de Bootstrap (Brown \& Rothery, 1993).

Los análisis de los parámetros productivos y las pérdidas causadas por la plaga se hicieron comparando los sistemas con gráficos de cajas y bigotes (Tukey, 1977; Hoaglin, 2003), análisis de varianza y comparaciones pareadas múltiples de Tukey para cada variable evaluada (Tukey, 1949; Duncan, 1955; Keselman \& Rogan, 1977; Tukey, 1977; Bland \& Altman, 1995; Hoaglin, 2003; Sawyer, 2009; McHugh, 2011). Los parámetros de fruta total y comercializable se usaron para estimar la pérdida asociada a la plaga en la semana 16 , cuando comenzó el crecimiento exponencial 
de la población de larvas. El Índice de Daño (ID) en los sistemas Comercial y Cero se calculó mediante regresión lineal en el periodo de linealidad en la curva de daño (Pedigo, Hutchins, \& Higley, 1986), excluyendo las plantas con producciones menores a $3500 \mathrm{~g} /$ planta (producción mínima asegurada para la variedad por la casa comercial de la semilla). Para los análisis se utilizó el programa PAST versión 3.22 (Hammer, Harper, \& Ryan, 2001).

\section{RESULTADOS}

Se registró una temperatura mínima y máxima de 7.4 y $33.3{ }^{\circ} \mathrm{C}$, respectivamente, con temperatura media entre 19.05 y $23.55^{\circ} \mathrm{C}$, y humedad relativa entre 31 y $98 \%$, con media entre 62.5 y $76.5 \%$. Los registros climáticos se presentan en la Tabla 1.

Las larvas de T. absoluta crecieron exponencialmente en el sistema Tuta a partir de la semana 14 , con un máximo en la semana 20 y una disminución acelerada en las semanas 21 a 25, para desaparecer con la muerte de las plantas (Fig. 1A). En el sistema comercial la población aumentó rápidamente a partir de la semana 19 (Fig. 1B), a pesar de los insecticidas aplicados, pero con un máximo muy inferior al alcanzado en el sistema sin control (Fig. 1A, 1B). En el tiempo, las larvas presentaron una mayor asociación a la sección superior de las plantas a partir de la semana 18 (Fig. 1C), aunque al comienzo fue mayor en la sección

TABLA 1

Resumen de variables climáticas en el ensayo

TABLE 1

Summary of climatic variables in the study

\begin{tabular}{lccc}
\multicolumn{1}{r}{ Parámetros } & Rango & Media ciclo & $\mathrm{DE}^{*}$ \\
$\mathrm{~T}^{\circ} \mathrm{C}$ mínima & $7.40-14.30$ & 12.00 & 1.751 \\
$\mathrm{~T}^{\circ} \mathrm{C}$ máxima & $25.70-33.30$ & 30.14 & 2.114 \\
$\mathrm{~T}^{\circ} \mathrm{C}$ media & $19.05-23.55$ & 21.07 & 1.220 \\
HR mínima (\%) & $31.00-57.00$ & 44.20 & 6.261 \\
HR máxima (\%) & $93.00-98.00$ & 94.90 & 1.213 \\
HR media (\%) & $62.50-76.50$ & 69.55 & 3.457 \\
\hline
\end{tabular}

* Desviación Estándar / Standard deviation. baja, hasta la semana 8 (Fig. 2A), luego en las secciones media y superior hasta la semana 17 (Fig. 2B) y en la superior después de la semana 18 (Fig. 2C). La sección apical tuvo la menor incidencia durante todo el cultivo (Fig. 2D). La captura de machos tuvo un crecimiento poblacional exponencial después de la semana 23, con un máximo de 953 adultos en la semana 27; las semanas anteriores fue fluctuante en el tiempo (Fig. 1D).

Para el ciclo completo del cultivo, los resultados se ajustaron mejor al modelo gaussiano $(\mathrm{AIC}=1.4599 \mathrm{E} 06)$ (Tabla 2), con un máximo poblacional de 584.41 larvas/planta en la semana 20, y una desviación estándar de 2.16 semanas (Fig. 1E), con un mínimo de 317 y un máximo de 802 larvas/planta en la semana 20. Los modelos que mejor describieron la dinámica de la población hasta la semana 19, justo antes de alcanzar el máximo poblacional, fueron el logístico y el exponencial (Tabla 3). No obstante, para evaluar el crecimiento de la población de larvas, se usaron los parámetros estimados en el modelo exponencial (Tabla 2), donde el parámetro "b" permite estimar una

TABLA 2

Parámetros de los modelos exponencial y gaussiano, describiendo el crecimiento poblacional de las larvas de $T$. absoluta en el sistema Tuta, con intervalos de confianza del $95 \%$ en paréntesis

TABLE 2

Parameters for Gaussian and exponential models, describing the larvae population growth of $T$. absoluta at the Tuta system, with $95 \%$ confidence intervals in parenthesis

\begin{tabular}{ccc} 
& Exponencial* & Gaussiano** \\
Fórmula & $\mathrm{N}(\mathrm{t})=\mathrm{ae}^{\mathrm{b} . \mathrm{t}}$ & $\mathrm{N}(\mathrm{t})=\mathrm{ae}\left((\mathrm{t}-\mathrm{b})^{2}\right) / 2 \mathrm{c}^{2}$ \\
$\mathrm{a}$ & $0.099(-0.201 ; 0.1826)$ & $584.41(523.3 ; 642.3)$ \\
$\mathrm{b}$ & $0.447(0.378 ; 0.504)$ & $20.093(19.91 ; 20.28)$ \\
$\mathrm{c}$ & $\mathrm{N} / \mathrm{A}$ & $2.046(1.843 ; 2.235)$ \\
\hline
\end{tabular}

\footnotetext{
* a, población inicial; $\mathbf{b}$, tasa intrínseca de crecimiento / a, initial population; $\mathbf{b}$, intrinsic growth rate.

** a, población máxima (pico poblacional); b, tiempo al pico; c, desviación estándar / maximum population (population peak); b, time to the peak; c, Standard deviation.
} 

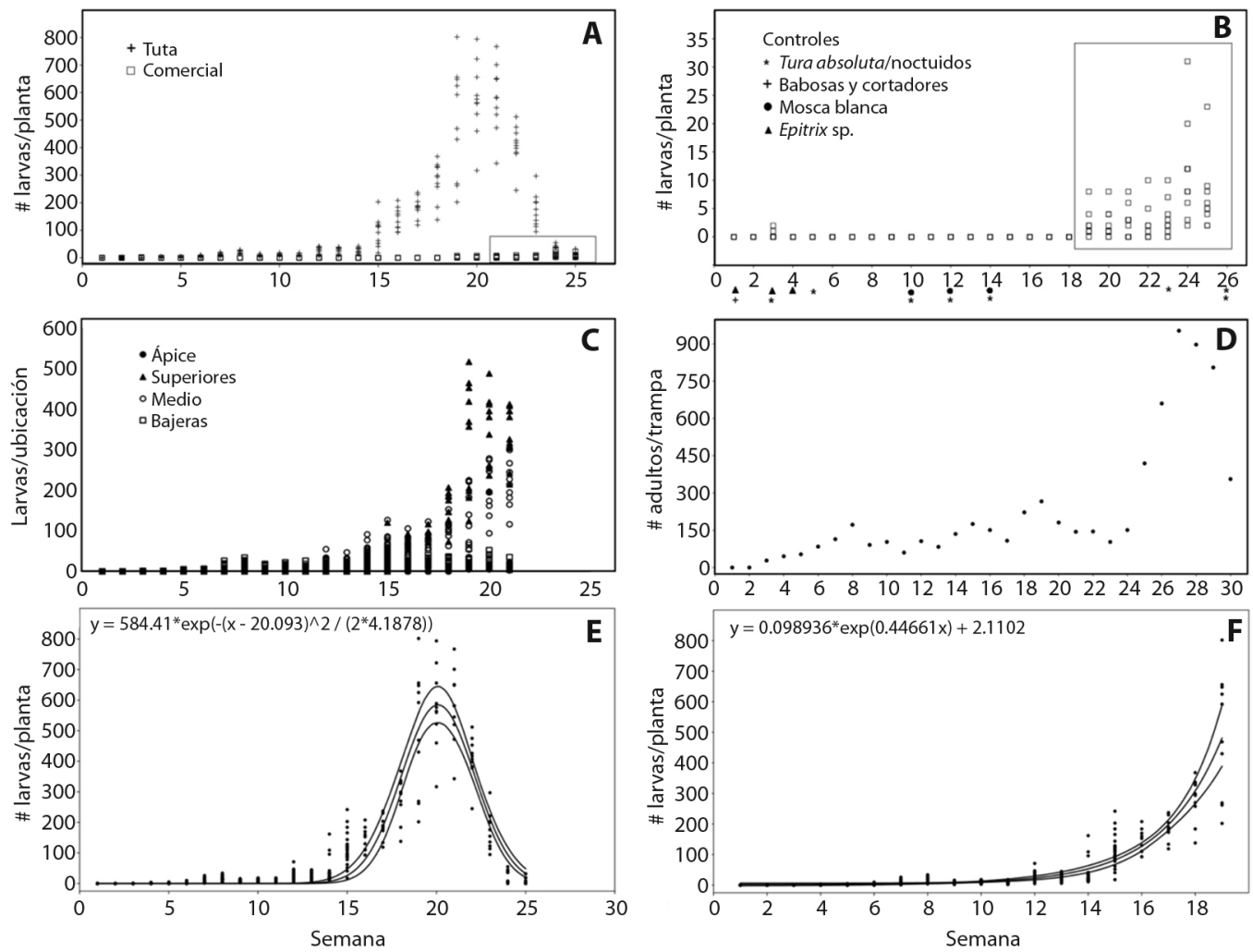

Fig. 1. Crecimiento poblacional de T. absoluta durante un ciclo completo de tomate de invernadero, con intervalos de confianza del $95 \%$. A. Número de larvas/planta para los sistemas productivos Tuta (máxima población) y comercial. B. Población en el sistema comercial; los símbolos bajo el eje X indican las semanas con aplicación de insecticidas y el objetivo de control. C. Cambios temporales y ubicación de larvas en la planta, para el sistema Tuta. D. Dinámica poblacional de machos capturados con feromona en el sistema comercial. E. Modelo de crecimiento poblacional gaussiano para larvas en el sistema Tuta; F. Modelo de crecimiento poblacional exponencial para larvas en el sistema Tuta en las semanas 1 a 19.

Fig. 1. Population growth of T. absoluta on a complete crop cycle of tomato under greenhouse conditions, with 95 $\%$ confidence intervals. A. The number of larvae/plant for the management systems Tuta (maximum population) and commercial. B. The larvae population at the commercial system; symbols under X-axis denote weeks with pesticide applications and pest objectives C. Temporal changes of larvae location in the plants at the Tuta system D. Population dynamics of males, caught by pheromones traps at the commercial system. E. Gaussian model for larvae population growth at the Tuta system $\mathbf{F}$. Exponential model for larvae population growth at the Tuta system through weeks 1 to 19.

tasa intrínseca de crecimiento poblacional de $0.4466(44.66 \%)$ por semana (Fig. 1F).

La pérdida de fruta en biomasa, y frutos totales y comercializables para los tres sistemas productivos se presenta en la Tabla 4. En el sistema Tuta (población máxima), las larvas afectaron el $100 \%$ del área foliar y causaron la muerte de las plantas y el fin de la producción en la semana 20. La producción de fruta total se redujo $38.62 \%$, y fue diferente significativamente comparada con el sistema comercial
$(\mathrm{P}=0.0002865)$, con 4250.75 a $2455.6 \mathrm{~g} /$ planta, pero sin diferencias con el sistema cero $(\mathrm{P}=0.0631)$ (Fig. 3A). No obstante, la diferencia no fue significativa entre los tres sistemas en el número de frutos por planta (Fig. 3B). Empero, la reducción de fruta comercializable fue significativa tanto en biomasa $(\mathrm{P}=2.48 \mathrm{E}-$ 08) (Fig. 3C), como en número de frutos $(\mathrm{P}=$ 1.28E-08) (Fig. 3D), con 4183.22 a 1000.40 $\mathrm{g} /$ planta, esto es, una pérdida de $74.73 \%$ entre el sistema comercial y el sistema Tuta. También 

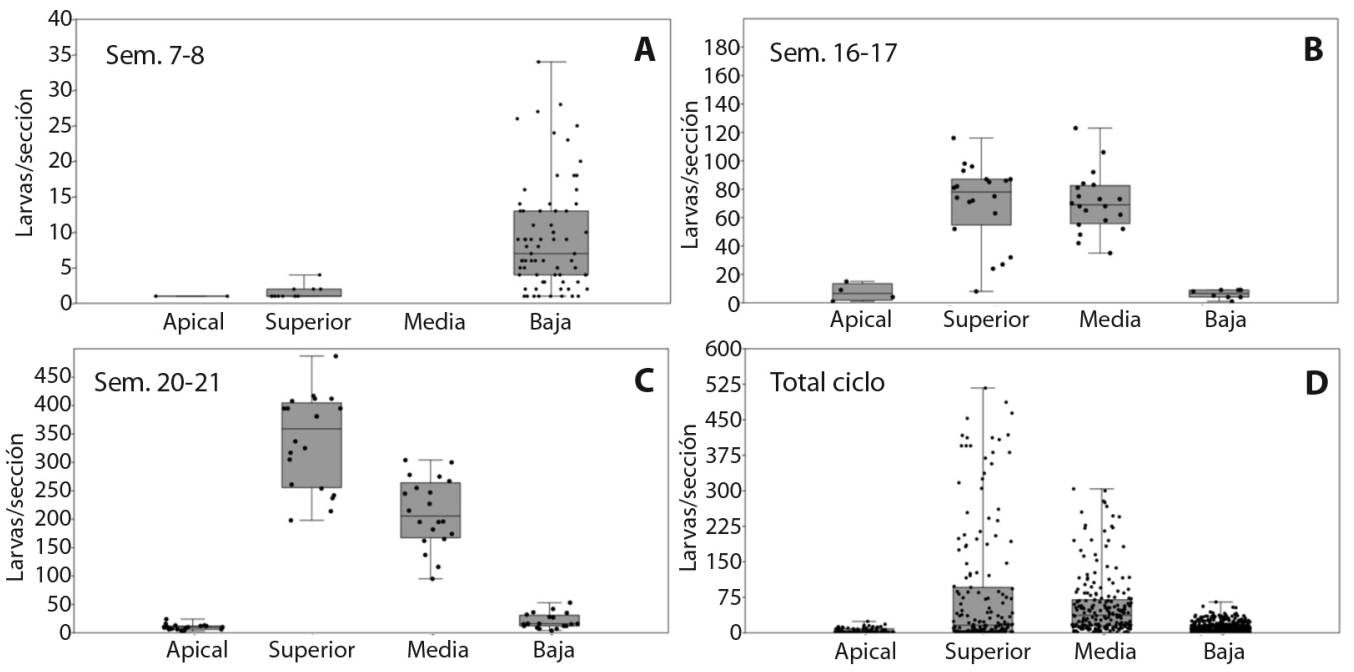

Fig. 2. Densidad de larvas de T. absoluta en partes del follaje en el sistema Tuta. A. semanas 7-8. B. semanas 16 - 17. C. semanas 20-21. D. ciclo total (semanas 1-21).

Fig. 2. Density of T. absoluta larvae in parts of the foliage in the Tuta system. A. weeks 7-8. B. weeks 16 - 17. C. weeks 20-21. D. complete crop cycle (weeks 1-21).

TABLA 3

Evaluación de los modelos descriptores de la población de larvas de T. absoluta, en 19 semanas de un cultivo de tomate bajo invernadero

TABLE 3

Evaluation of models describing the larvae population of T. absoluta in 19 weeks of a tomato crop under greenhouse conditions

\begin{tabular}{lccccc}
\multicolumn{1}{c}{ Modelo } & Fórmula & AIC & $\mathrm{a}$ & $\mathrm{b}$ & $\mathrm{c}$ \\
Lineal & $\mathrm{N}(\mathrm{t})=\mathrm{at}+\mathrm{b}$ & $2.35 \mathrm{E}+06$ & 12.881 & -70.991 & $\mathrm{~N} / \mathrm{A}$ \\
Cuadrático & $\mathrm{N}(\mathrm{t})=\mathrm{at}^{2}+\mathrm{bt}+\mathrm{c}$ & $1.15 \mathrm{E}+06$ & 2.292 & -30.027 & 71.127 \\
Potencia & $\mathrm{N}(\mathrm{t})=\mathrm{at}^{\mathrm{b}}+\mathrm{c}$ & $5.92 \mathrm{E}+05$ & $7.93 \mathrm{E}+08$ & 7.642 & 4.940 \\
Exponencial & $\mathrm{N}(\mathrm{t})=\mathrm{ae}^{\mathrm{b} . \mathrm{t}}$ & $\mathbf{5 . 8 4 E}+\mathbf{0 5}$ & 0.099 & 0.447 & $\mathrm{~N} / \mathrm{A}$ \\
Logístico & $\mathrm{N}(\mathrm{t})=\mathrm{a} /\left(1+\left(\mathrm{be} \mathrm{e}^{\mathrm{c} \cdot \mathrm{t}}\right)\right)$ & $\mathbf{5 . 7 9 E}+\mathbf{0 5}$ & -1007.2 & -3034.4 & 0.363 \\
Gaussian & $\mathrm{N}(\mathrm{t})=\mathrm{ae}\left((\mathrm{t}-\mathrm{b})^{2}\right) / 2 \mathrm{c}^{2}$ & $5.88 \mathrm{E}+05$ & $2.52 \mathrm{E}+06$ & 60.305 & 9.975 \\
\hline
\end{tabular}

Los valores para el mejor ajuste aparecen en negrita.

The values for goodness-of-fit of the best models in bold.

* Criterio de información de Akaike / Akaike Information Criterion.

hubo diferencias en el peso promedio del fruto entre los sistemas comercial y cero con el sistema Tuta ( $\mathrm{P}=0.01506)$, en el que los frutos fueron en promedio $23.61 \mathrm{~g}$ más livianos que en el sistema comercial. El sistema cero tuvo una producción menor al sistema comercial, en todos los parámetros evaluados. La reducción de fruta no comercializable en el sistema cero se debió a frutos de menor tamaño al exigido por el mercado. Para los demás parámetros evaluados (frutos por racimo, diámetro del tallo en el cuello y altura de las plantas), se encontraron diferencias significativas sólo en el número de racimos por planta, entre los sistemas Tuta y comercial (Fig. 3E), con un racimo más por planta en promedio, en este último. 

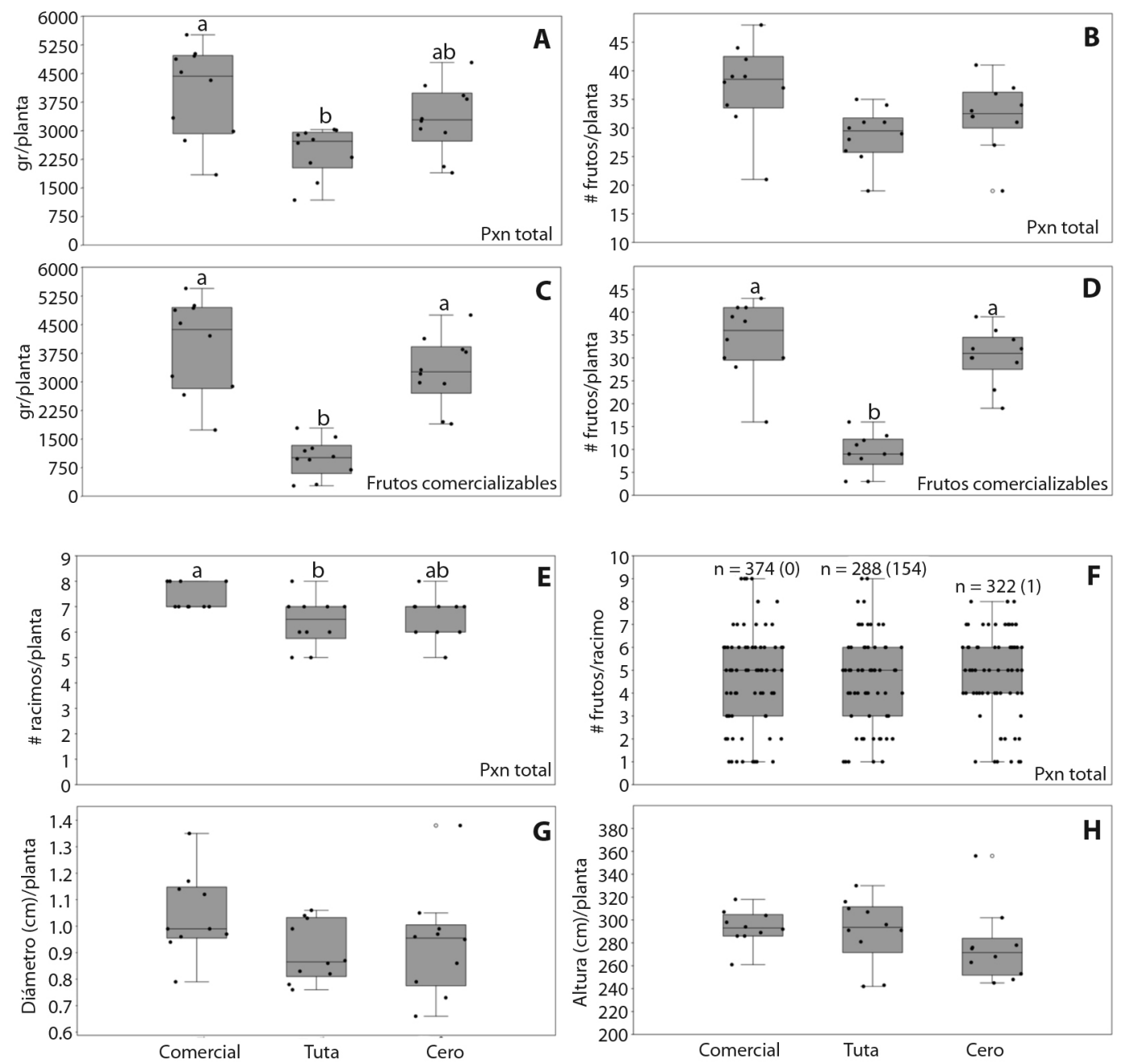

Fig. 3. Parámetros productivos de tomate de invernadero en los tres sistemas evaluados, Comercial, Tuta (máxima población) y Cero (población cero). Los círculos vacíos indican valores atípicos. A. producción total en g/planta. B. producción total de frutos por planta. C. producción total de frutos sanos en g/planta. D. frutos sanos por planta. E. producción en g/planta de frutos comercializables. F. frutos comercializables por planta. G. racimos por planta. H. frutos por racimo; en paréntesis se presenta el número total de frutos afectados no comercializables. I. diámetro del cuello de la planta. J. altura de las plantas. Fig. 3. Production parameters of greenhouse tomato in the three systems evaluated, Commercial, Tuta (maximum population) and Zero (total control). The empty circles indicate outliers. A. total production in g/plant. B. the total fruit production per plant. C. total production of healthy fruits in $\mathrm{g} /$ plant. D. healthy fruits per plant. E. production in $\mathrm{g} / \mathrm{plant}$ of marketable fruit. F. marketable fruit per plant. G. bunches per plant. H. fruit per bunch; the total number of affected notmarketable fruits in parenthesis. I. the stem plant diameter at the base. J. plant height.

Las plantas de los sistemas productivos presentaron promedios similares de frutos por racimo (Fig. 3F), aunque levemente mayores (un fruto más por racimo) en los sistemas comercial y cero, mientras que en el sistema Tuta fue de 4,06 frutos por racimo (Tabla 3). El diámetro de la planta en el cuello fue levemente mayor a $1 \mathrm{~cm}$ en el sistema comercial e inferior en los sistemas Tuta y cero (Fig. 3G). Finalmente, aunque la altura de las plantas (Fig. 3H) no presentó diferencias significativas, las plantas fueron más homogéneas en el sistema comercial 
TABLA 4

Parámetros productivos de tomate de invernadero en los tres sistemas asociados a niveles poblacionales de larvas de T. absoluta

TABLE 4

Production parameters of greenhouse tomato in the three systems, related to larvae population levels of T. absoluta

\begin{tabular}{lccc}
\multicolumn{1}{c}{ Parámetros/planta } & Comercial & Tuta & Cero \\
Producción total $(\mathrm{g})$ & $4250.75( \pm) 993.00$ & $2455.60( \pm) 634.70$ & $3321.30( \pm) 904.03$ \\
Producción comercializable $(\mathrm{g})$ & $4183.22( \pm) 1033.15$ & $1000.40( \pm) 486.96$ & $3277.10( \pm) 903.62$ \\
Frutos totales $(\#)$ & $37.40( \pm) 7.40$ & $28.80( \pm) 4.66$ & $32.20( \pm) 5.98$ \\
Frutos comercializables $(\#)$ & $34( \pm) 8.25$ & $9.30( \pm) 4.08$ & $30.40( \pm) 5.87$ \\
Peso promedio de frutos $(\mathrm{g})$ & $108.34( \pm) 22.65$ & $102.70( \pm) 18.70$ & $84.73( \pm) 15.04$ \\
Racimos (\#) & $7.40( \pm) 0.52$ & $6.40( \pm) 0.97$ & $6.60( \pm) 0.84$ \\
Frutos (\#)/racimo & $5.05( \pm) 2.27$ & $4.06( \pm) 1.89$ & $4.94( \pm) 1.94$ \\
Diámetro (cm) & $1.04( \pm) 0.16$ & $0.90( \pm) 0.11$ & $0.934( \pm) 0.20$ \\
Altura $(\mathrm{cm})$ & $293.5( \pm) 15.29$ & $290.7( \pm) 29.04$ & $276.4( \pm) 32.64$ \\
\hline
\end{tabular}

Desviación estándar en paréntesis. / Standard deviation in parenthesis.

TABLA 5

Valores de las regresiones lineales para la población de larvas y la biomasa total, biomasa comercializable, frutos totales y frutos comercializables

TABLE 5

Linear regression values for larvae population of $T$. absoluta and the total biomass, the marketable biomass, the total fruits, and the marketable fruits

\begin{tabular}{lcccc}
\multicolumn{1}{r}{ Parámetros } & Pendiente ID (IC 95 \%) & Intercepto (IC 95 \%) & Correlación (p) & $\mathrm{r}^{2}$ \\
Pxn. total $(\mathrm{g})$ & $-13696(-17312 ;-9$ 234) & $4490.6(4181.8 ; 4$ 845.8) & $-0.859(1.27 \mathrm{E}-06)$ & 0.74 \\
Pxn. comer. $(\mathrm{g})$ & $-22215(-26.12:-16$ 138) & $4342.2(3994.7 ; 4759.4)$ & $-0.911(2.33 \mathrm{E}-08)$ & 0.83 \\
\# frutos totales & $-0.068(-0.095 ;-0.040)$ & $38622(35711 ; 41563)$ & $-0.743(0.00017)$ & 0.81 \\
\# frutos comer. & $-0.175(-0.204 ;-0.135)$ & $35468(32.81 ; 38672)$ & $-0.912(2.16 \mathrm{E}-08)$ & 0.83 \\
\hline
\end{tabular}

* ID = Índice de daño; Pxn = producción.

(menor DE), lo que se asoció a un cultivo y una producción más uniforme.

Las regresiones lineales entre el número de larvas de Tuta y la biomasa indican un ID por planta de $13.70 \mathrm{~g}$ de fruta/larva para biomasa total (Fig. 4A). y de 22.22 gr fruta/larva para biomasa comercializable (Fig. 4B). En frutos comercializables (Fig. 4D), el total por planta fue 0.07 frutos/larva (Fig. 4C) y 0.18 frutos/ larva. Todas las regresiones fueron significativas y tuvieron valores de correlación $>0.70$ ) (Tabla 5).

El sistema Tuta tuvo una captura total de 8791 machos en los 30 días en la trampa, con máxima y mínima de 704 y 7 machos, respectivamente. La captura decreció exponencialmente en el tiempo, con una tasa intrínseca de -0.068 machos/día (Fig. 5).

\section{DISCUSIÓN}

En este estudio se evaluaron los parámetros poblacionales de $T$. absoluta en plantas de tomate en un cultivo comercial sin control de plagas. La tasa intrínseca de crecimiento semanal para la población de larvas fue 0.447 , con un marcado crecimiento exponencial después de la semana 15. La población alcanzó un 

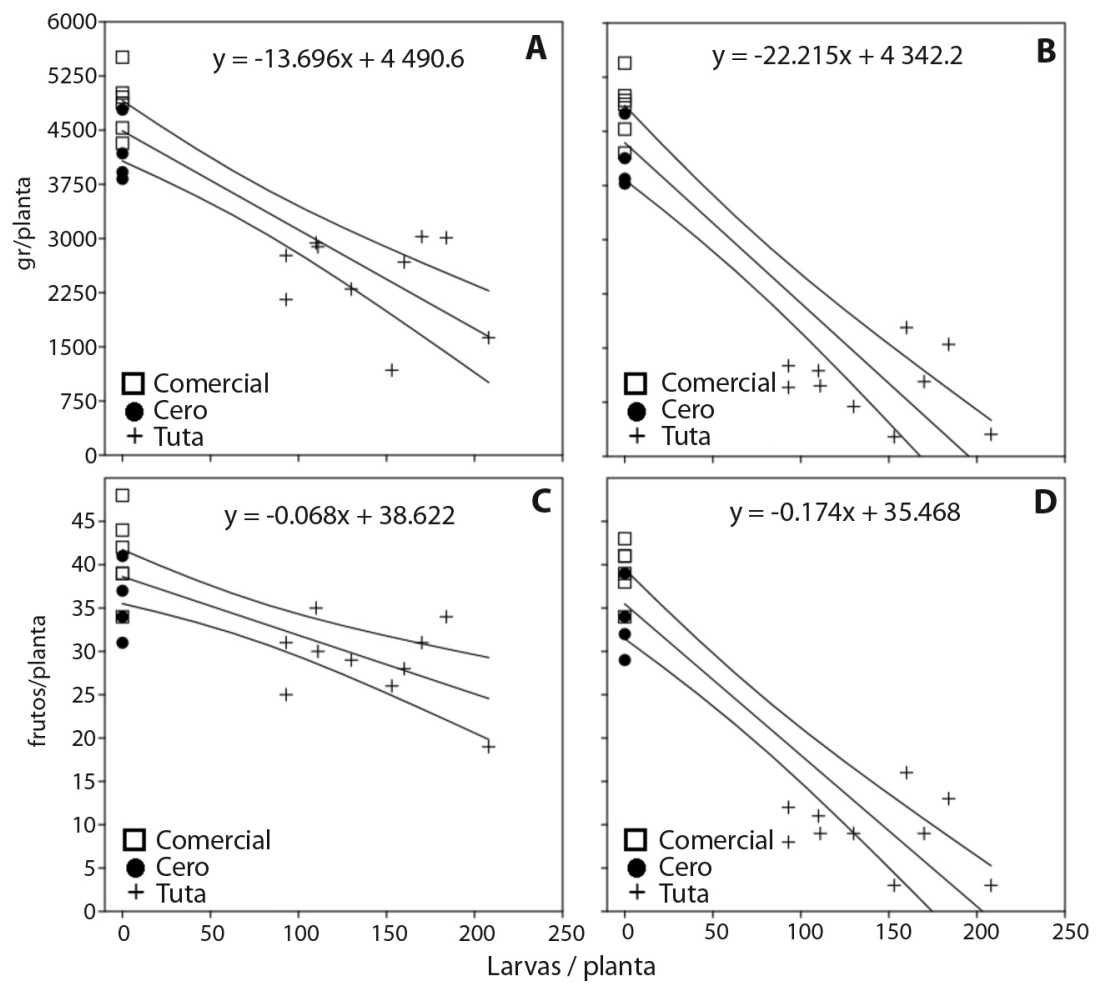

Fig. 4. Regresiones lineales para el índice de daño (ID) (pendiente) de larvas y la producción por planta de tomate, con intervalos de confianza del $95 \%$. A. g de fruta total. B. g de fruta comercializable. C. frutos totales. D. frutos comercializables.

Fig. 4. Linear regression for the Damage Index (DI) (slope) of larvae and production per tomato plant, with $95 \%$ confidence intervals. A. total fruit production (g). B. marketable fruit (g). C. number of total fruits. D. number of marketable fruits.

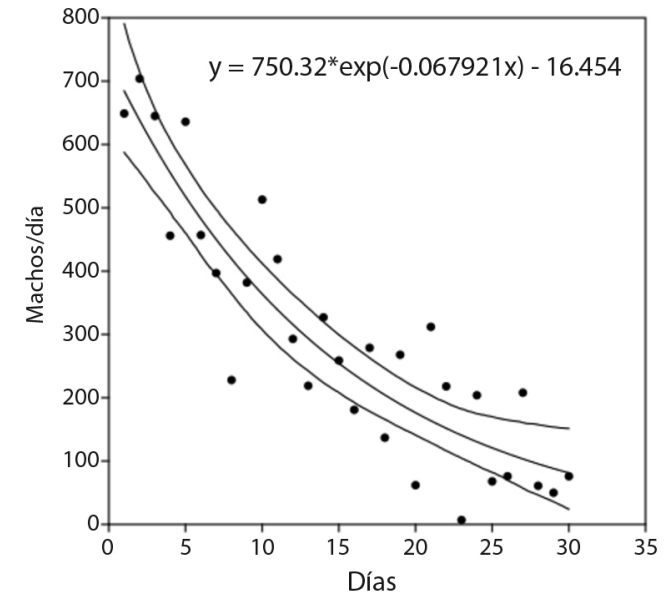

Fig. 5. Regresión no lineal para la disminución de machos de T. absoluta al término del ciclo del cultivo en el sistema Tuta.

Fig. 5. Non-linear regression for the decrease of males of T. absoluta at the end of the crop cycle in the Tuta system. promedio máximo de 584.4 larvas/planta en la semana 20, causando una disminución rápida del área foliar y la muerte de las plantas y el colapso del número de larvas en la semana 25. La tasa intrínseca de crecimiento calculada $(\approx$ 0.064 larvas/día), es casi la mitad de las encontradas en laboratorio sobre plantas de tomate en otros trabajos: $0.140 \pm 0.008$ /día a $29 \pm 0.5$ ${ }^{\circ} \mathrm{C}$ en Kanie Satishchandra et al., (2019); 0.14/ día a $25 \pm 1{ }^{\circ} \mathrm{C}$ en Pereyra y Sánchez (2006); $0.143 \pm 0.001 /$ día a $25.62 \pm 2{ }^{\circ} \mathrm{C}$ en Mohamadi Razmjou, Naseri y Hassanpour (2017); 0.300.346/día a $25 \pm 1{ }^{\circ} \mathrm{C}$ en Cekin y Yaşar (2015); y $0.132 /$ día a $25 \pm 1{ }^{\circ} \mathrm{C}$ en Erdogan y Babaroglu (2014). No obstante, esas estimaciones se hicieron mediante la construcción de tablas de vida y no en un cultivo bajo invernadero como en el presente estudio. 
Las diferencias encontradas se pueden deber a varios factores. El primero, que la temperatura media en el invernadero $\left(21.07{ }^{\circ} \mathrm{C}\right)$ fue inferior a las de cría en laboratorio mencionadas antes, por lo que se esperan ciclos de vida más largos (Cuthbertson et al., 2013). Para una media de $20^{\circ} \mathrm{C}$, cercana a la registrada en este trabajo, se encontraron ciclos de vida de 38.3 (Betancourt, Scatoni, \& Rodríguez, 1996) y 34.8 días (Krechemer \& Foerster, 2015), respectivamente. Ciclos más cortos generan más generaciones en el cultivo $\mathrm{y}$, por ello, una mayor tasa intrínseca de crecimiento. Por otra parte, en el cultivo puede ocurrir una alta mortalidad natural y disminuir la tasa intrínseca de crecimiento. Por ejemplo, Miranda Picanco, Zanuncio y Guedes (1998), registraron mortalidad natural de $92.3 \%$ para T. absoluta, desde huevo hasta adulto, sobre plantas de tomate en Brasil, y Cuthbertson et al., (2013) de $52 \%$ para condiciones de invernadero en el Reino Unido. Sin embargo, a una media de $30 \pm 5{ }^{\circ} \mathrm{C}$ bajo condiciones de invernadero abierto, Vivan Torres, Barros y Veiga (2002) encontraron para T. absoluta una tasa intrínseca de crecimiento de 0.061 individuos/día, lo que demuestra que los factores ambientales influyen en gran medida sobre la tasa de mortalidad en condiciones de campo.

Tang y Cheke (2008) estiman que los modelos matemáticos pueden apoyar en el diseño de estrategias de control y la toma de decisiones, ya que permiten aclarar y predecir los efectos de factores como los parasitoides, sobre las poblaciones plaga. En este trabajo, aunque el modelo gaussiano fue el mejor en describir la población de T. absoluta en todo el ciclo de cultivo, se utilizó el modelo exponencial para determinar la tasa intrínseca de crecimiento de la población plaga; conocer la velocidad de crecimiento poblacional de ésta serviría como modelo nulo para evaluar el efecto de medidas de manejo como el trampeo masivo o la liberación de parasitoides y depredadores sobre el desarrollo de la población, y sobre otros factores ambientales que podrían tener efecto sobre la misma (Horn, 2016). Asimismo, dicho parámetro poblacional permitiría estimar los tiempos y/o umbrales de acción en cultivos comerciales, aplicando la fórmula de crecimiento exponencial (ver métodos):

$$
\begin{aligned}
& \text { NDE (nivel de daño económico) }= \\
& \text { UA (umbral de acción) e } e^{r . t}, \\
& \text { donde: } \mathrm{N}(\mathrm{t})=\mathrm{NDE}, \mathrm{y} \mathrm{N}(0)=\mathrm{UA} \text {. }
\end{aligned}
$$

Por ejemplo, en Etiopía Shiberu y Getu (2018) determinaron que un UA de 3.82 larvas/planta y un NDE para T. absoluta de 2.87 larvas/planta; con la tasa de crecimiento aquí calculada $(r=0.4466)$, se puede obtener el tiempo de acción para evitar que la población llegue al NDE, así:

$$
3.82=2.87 \mathrm{e}^{0.4466 . t}
$$

despejando $\mathbf{t}$ de la fórmula: $\mathrm{t}=\ln (3.82 / 2.87) / 0.4466$;

$\mathrm{t}=0.6403$ semanas, es decir. $\approx 4.48 \mathrm{~d}$.

Así, definiendo el NDE en términos económicos, como sugieren Pedigo et al., (1986), el UA se podría definir en términos del potencial de incremento poblacional.

En términos productivos, comprender la dinámica de las plagas tiene sentido en la medida que permita estimar las pérdidas causadas y mejorar la toma de decisiones en campo. Para T. absoluta en tomate se ha evaluado el ID en diversas variedades y países. Por ejemplo, Ghaderi, Fathipour, Asgari y Reddy, (2019) encontraron por regresión en Irán ID por planta de 30.32 y $26.48 \mathrm{~g}$ de fruta/larva para los cultivares 'Cal JN3' y 'Early Urbana Y' mediante infestación artificial. En Colombia, en tomate 'Durinta' bajo invernadero, Cely, Cantor y Rodríguez (2010) encontraron un ID por planta de 0.85 frutos totales/larva y 1.15 frutos sanos/larva. En nuestro estudio, se estimaron pérdidas de $22.2 \mathrm{~g} \mathrm{y} 0.18$ de frutos totales y comercializables por larva/planta. Aunque en la producción de biomasa los valores son similares, la producción en número de frutos presenta diferencias importantes. Sin embargo, es de notar que el ID calculado por Cely et al., (2010) se hizo con un menor número de plantas. Así, con nuestros resultados, para una población 
de 100 larvas/planta en la semana de inicio de cosecha (16), se espera una reducción de 2222 $\mathrm{g}$ por planta. Con este valor se pueden ajustar los NDE en sistemas productivos de tomate similares.

Sridhar, Chakravarthy y Asokan (2014), definieron en la India que 0.08 galerías de $T$. absoluta/planta causaron un daño bajo y 14.08 galerías/planta un daño alto, con un máximo de 30 a 40 galerías/planta, y sugirieron que la plaga prefiere infestar los brotes apicales y tiernos del tomate, afectando el $87 \%$ de las plantas y causando $3.5 \%$ de frutos dañados. Kumari et al., (2015) y Harbi, Abbes y Chermiti (2012), describieron los daños en 14.4 y $97 \%$ de hojas dañadas, respectivamente para todo el ciclo del cultivo. Nuestro trabajo indica que el mayor daño se debe a la perforación de frutos, que causa perdidas en fruta comercializable de $74.73 \%$, y no por disminución de la cantidad de frutos por planta. No obstante, los daños foliares producidos por las larvas acortaron el ciclo productivo de la planta en 5 semanas, y probablemente se asoció a un menor llenado de frutos. Así pues, el efecto de la plaga perforando frutos fue más importante que dañando brotes apicales y racimos florales.

La densidad de larvas en las secciones de la planta varió en el tiempo. Los resultados indican que la plaga se debe muestrear en el tercio inferior de la planta en las primeras semanas ( $<10$ después del trasplante) y en el tercio medio y superior después de la semana 15. Esto concuerda con Leite, Picanço, Silva, Mata y Jham (1995), quienes encontraron una menor preferencia por la parte apical, en variedades de tomate silvestre y con Cely et al., (2010) para una variedad comercial, pero contrasta con Torres, Faria, Evangelista y Pratissoli (2001), quienes encontraron mayor postura en las partes apicales y medias de la planta. Esto podría contribuir a mejorar los métodos de detección de la plaga en invernadero según el desarrollo de las plantas.

Para la detección de machos, la feromona sexual de T. absoluta se utiliza para localizar y monitorear las poblaciones (Guedes, \& Picanço, 2012), y también para el control de la plaga (Caparros Megido, Haubruge, \& Verheggen, 2013). Se ha sugerido que el recuento en la trampa debe ser semanal para seguir la evolución de los machos (Bolckmans, 2009), ya que el número capturado refleja el incremento en la población de larvas y, por tanto, en la producción de tomate (Monserrat-Delgado, 2008). Para el sistema Tuta, la captura total de machos registrada (8791 individuos) sugiere una población final de 17582 individuos, asumiendo una razón sexual de 1:1. Esto supondría un promedio final de 439.55 larvas en cada una de las 40 plantas. No obstante, si usara la proporción sexual indicada por Kanle-Satishchandra, Chakravarthy, Özgökçe y Atlihan (2019), donde las hembras fueron $67 \%$ de los individuos criados sobre tomate, la población total estimada sería de 26639 individuos, es decir, un promedio de 665.978 larvas por planta que completaron su ciclo de vida. Para Stol, Griepink y van Deventer (2009), 1 a 3 machos capturados por semana implican un riesgo de infestación bajo; 4 a 30 uno moderado $\mathrm{y}>30$ uno alto. Desneux (2010) reportó en Brasil y Chile capturas promedio de 19.50 y 100 machos/d, respectivamente. Finalmente, debe notarse que la tasa intrínseca de decrecimiento de la población de machos al final del cultivo fue muy similar a la tasa intrínseca de crecimiento estimada para las larvas.

El minador T. absoluta es la mayor amenaza para la producción de tomate en todo el mundo, tanto en zonas endémicas como en las de reciente invasión. Las limitadas herramientas disponibles para predecir la progresión de la plaga en los cultivos, y la toma de decisiones para su manejo, generan el uso excesivo de moléculas de síntesis química para su control, y consecuentemente la aparición de múltiples casos de resistencia a insecticidas. El desarrollo de modelos poblacionales, basados en el potencial reproductivo de la especie bajo condiciones de campo, podría refinar el cálculo de los umbrales de acción y los programas de manejo integrado, para las diferentes regiones o sistemas productivos. En este trabajo, se presenta el análisis de una población de T. absoluta bajo condiciones de cultivo, que, en las condiciones 
de invernadero evaluadas, tuvo un crecimiento exponencial, con tasa intrínseca de crecimiento de 0.447. El ID se calculó en $22.22 \mathrm{~g}$ de fruta comercializable/planta/larva y de 0.18 frutos comercializables/planta/larva.

Declaración de ética: los autores declaran que todos están de acuerdo con esta publicación y que han hecho aportes que justifican su autoría; que no hay conflicto de interés de ningún tipo; y que han cumplido con todos los requisitos y procedimientos éticos y legales pertinentes. Todas las fuentes de financiamiento se detallan plena y claramente en la sección de agradecimientos. El respectivo documento legal firmado se encuentra en los archivos de la revista.

\section{AGRADECIMIENTOS}

Los autores agradecen a Sonia del Carmen Jaramillo Villegas y Luis Hernán Gonzalez Santamaría por disponer el predio y financiar este trabajo. La publicación de este trabajo fue financiada por la dirección de Investigación y Desarrollo de la Universidad Católica de Oriente.

\section{RESUMEN}

Introducción: El minador de hojas de tomate, Tuta absoluta (Meyrick), la plaga más peligrosa para la producción de tomate, Solanum lycopersicum L., en todo el mundo; puede causar pérdidas totales y es difícil de controlar. Por lo tanto, comprender la dinámica de sus poblaciones en el cultivo es una prioridad para mitigar el daño. Objetivo: Aquí, los parámetros de la población de T. absoluta fueron evaluados en tomate var. Sonero en invernadero, en tres sistemas de gestión (comercial, población máxima y control total). Métodos: durante 25 semanas en La Unión, Antioquia, Colombia, se aplicaron modelos matemáticos para describir las poblaciones de larvas y adultos (machos) en el tiempo, en relación con los parámetros de producción y las pérdidas asociadas. Resultados: La tasa de crecimiento intrínseco fue de 0.4466 larvas por semana. Los modelos gaussianos y exponenciales describieron mejor a la población en el tiempo. Las pérdidas se asociaron con el peso y la cantidad de frutos comercializables, y también con el peso total de producción y la cantidad de racimos y frutos totales. Conclusión: Las larvas de T. absoluta tienen un crecimiento exponencial en invernadero ventilado, con una tasa de crecimiento intrínseca menor que la calculada por las tablas de vida en el laboratorio.

Palabras claves: Índice de daño; minador del tomate; polilla del tomate; Manejo Integrado de Plagas; crecimiento exponencial.

\section{REFERENCIAS}

Araya, J.E., Hernando, S., \& Fereres, A. (2017). Algunos enemigos naturales de interés para la polilla del tomate, Tuta absoluta. En Memorias Cientificas del Congreso Internacional de Agricultura Sustentable. Ecuador: Centro de Investigación y Desarrollo.

Balzan, M.V., \& Moonen, A.C. (2012). Management strategies for the control of Tuta absoluta (Lepidoptera: Gelechiidae) damage in open-field cultivations of processing tomato in Tuscany (Italy). EPPO Bulletin, 42(2), 217-225.

Betancourt, C.M., Scatoni, I.B., \& Rodríguez, J.J. (1996). Influencia de la temperatura sobre la reproducción y el desarrollo de Scrobipalpuloides absoluta (Meyrick) (Lepidoptera. Gelechiidae). Revista Brasileira de Biología, 56, 661-670.

Bland, J.M., \& Altman, D.G. (1995). Tukey Multiple Comparison test. British Medical Journal of Clinical Nursing, 310(8), 299-304.

Brown, D., \& Rothery, P. (1993). Models in biology: mathematics, statistics and computing. Chichester, Reino Unido: John Wiley \& Sons Ltd.

Caparros Megido, R., Haubruge, E., \& Verheggen, F. (2013). Pheromone-based management strategies to control the tomato leafminer Tuta absoluta (Lepidoptera: Gelechiidae). A review. Biotechnologie, Agronomie, Société et Environnement, 17(3), 475-482.

Cely, P., Cantor, F., \& Rodríguez, D. (2010). Determination of levels of damage caused by different densities of Tuta absoluta populations (Lepidoptera: Gelechiidae) under greenhouse conditions. Agronomía Colombiana, 28(3), 392-402.

Cocco, A., Serra, G., Lentini, A., Deliperi, S., \& Delrio, G. (2015). Spatial distribution and sequential sampling plans for Tuta absoluta (Lepidoptera: Gelechiidae) in greenhouse tomato crops. Pest Management Science, 71, 1311-1323.

Cook, R.D. (1977). Detection of Influential Observation in Linear Regression. Technometrics, 19(1), 15-18. DOI: $10.1080 / 00401706.1977 .10489493$

Cuthbertson, A.G., Mathers, J.J., Blackburn, L.F., Korycinska, A., Luo, W., Jacobson, R.J., \& Northing, P. (2013). Population development of Tuta absoluta (Meyrick) (Lepidoptera: Gelechiidae) under simulated UK glasshouse conditions. Insects, 4(2), 185-197. 
Desneux, N., Wajnberg, E., Wyckhuys, K.A., Burgio, G., Arpaia, S., Narváez-Vasquez, C.A., \& Pizzol. J. (2010). Biological invasion of European tomato crops by Tuta absoluta: ecology, geographic expansion and prospects for biological control. Journal of Pest Science, 83(3), 197-215.

Duncan, D.B. (1955). Multiple Range and Multiple F Tests. Biometrics, 11(1), 1-42. DOI: $10.2307 / 3001478$

Erdogan, P., \& Babaroglu, N.E. (2014). Life table of the tomato leaf miner Tuta absoluta (Meyrick) (Lepidoptera: Gelechiidae). Journal of Agricultural Faculty of Gaziosmanpasa University, 31, 80-89.

FAO. (2016). Management of Tomato Borer: Tuta absoluta in Near East Region. Recuperado de http:// www.fao.org/lebanon/programmes-and-projects/ success-stories/ipm/en

Flores, J., Ojeda-Bustamante, W., López, I., Rojano, A., \& Salazar, I. (2007). Requerimientos de riego para tomate de invernadero. Terra Latinoamericana, 25(2), 127-134.

Franceschetti, G., \& Riccio, D. (2007). Scattering. natural surfaces. and fractals. San Diego, Estados Unidos de América: Elsevier.

Ghaderi, S., Fathipour, Y., Asgari, S., \& Reddy, G.V. (2019). Economic injury level and crop loss assessment for Tuta absoluta (Lepidoptera: Gelechiidae) on different tomato cultivars. Journal of Applied Entomology, 143(5), 493-507.

Goftishu, M., Seid, A., \& Dechassa, N. (2014). Occurrence and population dynamics of tomato leaf miner [Tuta absoluta (Meyrick). Lepidoptera: Gelechiidae] in Eastern Ethiopia. East African Journal of Sciences, $8(1), 59-64$.

González, C.G., Lise, A.V., \& Felpeto, A.B. (2013). Tratamiento de datos con R, Statistica y SPSS. Madrid, España: Ediciones Díaz de Santos.

Guedes, R.N.C., \& Picanço, M.C. (2012). The tomato borer Tuta absoluta in South America: pest status. management and insecticide resistance. EPPO Bulletin, 42(2), 211-216.

Guimapi, R.Y., Mohamed, S.A., Okeyo, G.O., Ndjomatchoua, F.T., Ekesi, S., \& Tonnang, H.E. (2016). Modeling the risk of invasion and spread of Tuta absoluta in Africa. Ecological Complexity, 28, 77-93. DOI: 10.1016/j.ecocom.2016.08.001

Hammer, Ø., Harper, D.A., \& Ryan, P.D. (2001). PAST: Paleontological statistics software package for education and data analysis. Palaeontologia Electronica, 4(1), 1-9. DOI: 10.1016/j.bcp.2008.05.025

Harbi, A., Abbes, K., \& Chermiti, B. (2012). Evaluation of two methods for the protection of tomato crops against the tomato leafminer Tuta absoluta (Meyrick) under greenhouses in Tunisia. EPPO Bulletin, 42(2). 317-321.

Hoaglin, D.C. (2003). John W. Tukey and Data Analysis. Statistical Science, 18(3), 311-318. DOI:10.1214/ ss/1076102418

Holdridge, L.R. (1967). Life zone ecology. San José, Costa Rica: Tropical Science Center.

Horn, D.J. (2016). Ecological control of insects. Boca Ratón, Estados Unidos de América: CRC Press.

Kanle-Satishchandra, N., Chakravarthy, A.K., Özgökçe, M.S., \& Atlihan, R. (2019). Population growth potential of Tuta absoluta (Meyrick) (Lepidoptera: Gelechiidae) on tomato. potato. and eggplant. Journal of Applied Entomology, 143(5), 518-526.

Keselman, H.J., \& Rogan, J.C. (1977). The Tukey multiple comparison test: 1953-1976. Psychological Bulletin, 84(5), 1050-1056.

Krechemer, F.D.S., \& Foerster, L.A. (2015). Tuta absoluta (Lepidoptera: Gelechiidae): Thermal requirements and effect of temperature on development, survival, reproduction and longevity. EJE, 112(4), 658-663.

Kumari, D.A., Anitha, G., Anitha, V., Lakshmi, B.K.M., Vennila, S., \& Rao, N.H.P. (2015). New record of leaf miner, Tuta absoluta (Meyrich) in Tomato. Insect Environment, 20(4), 136-138.

Leite, G.L.D., Picanço, M., Silva, D.D., Mata, A.D., \& Jham, G.N. (1995). Distribuição de oviposição de Scrobipalpuloides absoluta no dossel de Lycopersicon esculentum. L. hirsutum e L. peruvianum. Horticultura Brasilera, 13, 47-51.

Marchioro, C.A., Krechemer, F.S., \& Foerster, L.A. (2017). Estimating the development rate of the tomato leaf miner. Tuta absoluta (Lepidoptera: Gelechiidae). using linear and non-linear models. Pest Management Science, 73(7), 1486-1493. DOI:10.1002/ps.4484

McHugh, M.L. (2011). Multiple comparison analysis testing in ANOVA. Biochemia Medica, 21(3), 203-209.

Miranda, M.M.M., Picanco, M., Zanuncio, J.C., \& Guedes, R.N.C. (1998). Ecological life table of Tuta absoluta (Meyrick) (Lepidoptera: Gelechiidae). Biocontrol Science and Technology, 8(4), 597-606.

Mohamadi, P., Razmjou, J., Naseri, B., \& Hassanpour, M. (2017). Population Growth Parameters of Tuta absoluta (Lepidoptera: Gelechiidae) on Tomato Plant Using Organic Substrate and Biofertilizers. Journal of Insect Science, 17(2), 1-7.

Mohamed, E.S.I., Mohamed, M.E., \& Gamiel, S.A. (2012). First record of the tomato leafminer. Tuta absoluta (Meyrick) (Lepidoptera: Gelechiidae) in Sudan. EPPO Bulletin, 42(2), 325-327. 
Nguyen, H.D.D., \& Nansen, C. (2018). Edge-biased distributions of insects. A review. Agronomy for sustainable development, 38(1), 1-11.

Özgökçe, M.S., Bayindir, A., \& Karaca, I. (2016). Temperature-dependent development of the tomato leaf miner Tuta absoluta (Meyrick) (Lepidoptera: Gelechiidae) on tomato plant Lycopersicon esculentum Mill (Solanaceae). Türkiye Entomoloji Dergisi, 40(1), 51-59.

Pedigo, L.P., Hutchins, S.H., \& Higley, L.G. (1986). Economic injury levels in theory and practice. Annual Review of Entomology, 31(1), 341-368.

Pereyra, P.C., \& Sánchez, N.E. (2006). Effect of two solanaceous plants on developmental and population parameters of the tomato leaf miner. Tuta absoluta (Meyrick) (Lepidoptera: Gelechiidae). Neotropical Entomology, 35(5), 671-676.

Pihán, R., \& Marin, C. (2000). Producción de hortalizas de fruto bajo plástico. Programa de capacitación para la producción hortofrutícola en Angol y Renaico. Boletín INIA, 32.

Rencher, A.C., \& Schaalje, G.B. (2008). Linear models in statistics. Chichester, Reino Unido: John Wiley \& Sons Ltd.

Roditakis, E., Vasakis, E., García-Vidal, L., del Rosario Martínez-Aguirre, M., Rison, J.L., Haxaire-Lutun, M.O., \& Bielza, P. (2018). A four-year survey on insecticide resistance and likelihood of chemical control failure for tomato leaf miner Tuta absoluta in the European/Asian region. Journal of Pest Science, 91(1), 421-435.

Safer Agrobiológicos. (2017). Feromona para Tuta absoluta - Ficha técnica. Recuperado de file://E:/Users/ Usuario/Downloads/F.T\%20Feromona\%20Tuta.pdf

Salazar, E.R., \& Araya, J.E. (2001). Tomato moth Tuta absoluta (Meyrick) response to insecticides in Arica. Agricultura Técnica, 61 (4), 429-435.

Sawyer, S.F. (2009). Analysis of Variance: The Fundamental Concepts. Journal of Manual \& Manipulative Therapy, 17(2), 27E-38E. DOI: 10.1179/ jmt.2009.17.2.27e

Sharma, Y., Abbott, K.C., Dutta. P.S., \& Gupta, A.K (2015). Stochasticity and bistability in insect outbreak dynamics. Theoretical Ecology, 8(2), 163-174.

Shiberu, T., \& Getu, E. (2018). Experimental analysis of ecomomic action level of tomato leafminer. Tuta absoluta Meyrick (Lepidoptera: Gelechiidae) on tomato plant under open field. Advances in Crop Science and Technology, 6(1), 1-5.

Silva, W.M., Berger, M., Bass, C., Balbino, V.Q., Amaral, M.H., Campos, M.R., \& Siqueira, H.A. (2015). Status of pyrethroid resistance and mechanisms in Brazilian populations of Tuta absoluta. Pesticide Biochemistry and Physiology, 122, 8-14.

Sridhar, V., Chakravarthy, A.K., \& Asokan, R. (2014). New record of the invasive South American tomato leaf miner, Tuta absoluta (Meyrick) (Lepidoptera: Gelechiidae) in India. Pest Management in Horticultural Ecosystems, 20(2), 148-154.

Stol, W., Griepink, F.C., \& van Deventer, P. (2009). Tuta absoluta: una nueva plaga para el cultivo del tomate en Europa. En II Jornadas sobre Feromonas, atrayentes, trampas y Control Biológico: Alternativas para la agricultura del siglo XXI, Murcia, Spain, 2009-11-18/2009-11-19. España.

Tang, S., \& Cheke, R.A. (2008). Models for integrated pest control and their biological implications. Mathematical Biosciences, 215(1), 115-125.

Torres, J.B., Faria. C.A., Evangelista, W.S., \& Pratissoli, D. (2001). Within-plant distribution of the leaf miner Tuta absoluta (Meyrick) immatures in processing tomatoes. with notes on plant phenology. International Journal of Pest Management, 47(3), 173-178. DOI: $10.1080 / 02670870010011091$

Tukey, J.W. (1949). Comparing individual means in the analysis of variance. Biometrics, 5(2), 99-114. DOI: $10.2307 / 3001913$.

Tukey, J.W. (1977). Exploratory data analysis. Reading, Estados Unidos de América: Addison-Wesley.

van der Straten, M.J.J., Potting, R.P.J., \& van der Linden, A. (2011). Introduction of the tomato leafminer Tuta absoluta into Europe. Proceedings of the Netherlands Entomological Society Meeting, 22, 23-30.

Vandermeer, J. (2010). How populations grow: the exponential and logistic equations. Nature Education Knowledge, 3(10), 1-15.

Vivan, L.M., Torres. J.B., Barros, R., \& Veiga, A. (2002). Tasa de crecimiento poblacional del chinche depredador Podisus nigrispinus (Heteroptera: Pentatomidae) y de la presa Tuta absoluta (Lepidoptera: Gelechiidae) en invernadero. Revista de Biología Tropical, $50(1), 145-153$.

Zekeya, N., Chacha, M., Ndakidemi, P., Materu, C., Chidege, M., \& Mbega, E. (2017a). Tomato Leafminer (Tuta absoluta Meyrick 1917): A Threat to Tomato Production in Africa. Journal of Agriculture and Ecology Research International, 10(1), 1-10. DOI: 10.9734/JAERI/2017/28886

Zekeya, N., Ndakidemi, P.A., Chacha, M., \& Mbega, E. (2017b). An emerging agricultural pest in SubSaharan Africa: Current and prospective management strategies. African Journal of Agricultural Research, 12(6), 389-396. DOI: 10.5897/AJAR2016.11515 